\title{
What makes children defy their peers? Chinese and Spanish preschoolers' decisions to trust (or not) peer consensus
}

\section{Carla Sebastián-Enesco $^{1}$ (D) | Silvia Guerrero ${ }^{2}$ | Ileana Enesco ${ }^{3}$}

\begin{abstract}
The more people agree on the same piece of information, the more likely are individuals to endorse the testimonial information. Children are sensitive to consensus but their trust in what a majority says also depends on the decision context, their previous knowledge, and, interestingly, the culture in which they develop. Here we study Chinese $(N=60)$ and Spanish $(N=48)$ preschoolers' sensitivity to the opinion of a group of peers in consensus regarding (a) peer interaction events and (b) use of artifacts. For each context, we varied the degree of ambiguity of the situations: unfamiliar, ambiguous, and familiar, where the majority offered a transgressive opinion in conflict with "common sense." Children were more likely to trust their peers in the unfamiliar and ambiguous situations. In the familiar situations, children showed greater acceptance of transgressive claims regarding artifacts than peer interaction events. The two cultural groups, however, significantly differed in the degree of endorsement. Although Chinese children gave little credibility to their peers even when facing novel information, Spaniards deferred to them, even at the expense of their own criteria. Together with previous findings, these results indicate culture-specific patterns related to children's attitudes toward peers versus adults as sources of knowledge.

KEYWORDS

children, culture-comparative, peer consensus, testimonial learning, trust
\end{abstract}




\section{1 | INTRODUCTION}

One of the keys to the success of human species is the capacity to acquire knowledge from the testimony of others. This type of social learning is at the root of the process of cultural accumulation, which takes place from one generation to another, through the history of humanity (Tomasello, 1999; see also Claidière \& Whiten, 2012 for a comprehensive review in humans and non-humans). Although humans, like other animal species, build knowledge through their own experience and actions in the physical and social worlds, the vast majority of what we know comes from what others say or write, either because this information is not accessible firsthand (e.g., Who was Aristotle?) or because it is burdensome to verify directly (e.g., What is the molecular structure of water?). An essential condition for this type of learning to prosper is the credibility an individual gives to the testimony of others. For example, there is a difference between acquiring a piece of information from an expert in an academic context and from friends who lack specialized knowledge during a chat over coffee.

Over the last 15 years, there has been a growing interest in the study of trust in the testimony of others during childhood (for a recent review, see Harris, Koenig, Corriveau, \& Jaswal, 2018). A key finding of the recent research is that, contrary to general belief, children do not blindly trust others' testimony, but they weigh a series of factors when deciding whether to accept information provided by others. In general terms, these factors can be divided into three broad, interrelated categories: the characteristics of the informant/s (including the number of informants), the domain of knowledge, and the individual's prior knowledge of the topic in question. However, the influence of these factors on children's likelihood of accepting testimonial information is modulated by the culture in which individuals develop. Although most studies to date have been conducted with children from industrialized Western societies, especially from the United States, the few studies carried out in other countries suggest the existence of cultural variation in the endorsement of others' testimony (Chan \& Tardiff, 2013; Corriveau, Fusaro, \& Harris, 2013), especially when a majority provides testimony that contradicts the child's prior knowledge. In the present study, we examined whether children from two cultural backgrounds, Spain and China, were equally likely to endorse-or reject-the testimony provided by a group of peers in consensus. Crucially, the testimonial information presented sometimes ran against the children's prior ideas.

One of the most studied aspects of informants is the number of people who provide the same information, and in particular, whether there exists a unanimous consensus, or by contrast, whether the informants offer different opinions. Studies report that, similar to adults (Bond \& Smith, 1996), children, from at least the age of four, take into account the number of people who agree on the same information, and are more willing to accept this information from a consensus majority than from a partial majority, that is, when there are dissident opinions (Bernard, Harris, Terrier, \& Clément, 2015; Chen, Corriveau, \& Harris, 2013; Corriveau, Fusaro, \& Harris, 2009; Guerrero, Cascado, Sausa, \& Enesco, 2017; Morgan, Laland, \& Harris, 2015). In other words, children use the degree of consensus on a piece of information to make inferences about the veracity of this information.

Nonetheless, the influence of consensus information greatly depends on the child's prior knowledge of the subject. When faced by novel information, most children endorse the majority view (Corriveau et al., 2009; Einav, 2014). The classic example is the learning of new words, where the information transmitted by testimony is presumably neutral with respect to the individual's knowledge. The most commonly used experimental situation consists of presenting a new object for which a series of informants (normally adults) provide a name, this usually being a pseudoword (e.g., "reso" to refer to a novel artifact). In this situation, children choose the label proposed by the majority in around $65 \%$ of occasions rather than the label proposed by a dissenter (e.g., "tero") (Corriveau et al., 2009; but see Schillaci \& Kelemen, 2014 for different results in the context of novel objects' function).

However, when children possess prior knowledge or beliefs about the situation at hand and a majority of informants contradict this information, they are more reluctant to endorse the majority view (Einav, 2014). An extreme case is that of the classic experiment designed by Asch (1956), in which a group of informants contradicted the participant's visual experience (e.g., identifying the longest of a set of lines). In this situation, less than 30\% of 4-5year olds sided with a peer majority whose perceptive judgment was clearly incorrect (Haun \& Tomasello, 2011). 
Unsurprisingly, children, in the same way as adults (Bond \& Smith, 1996), give more weight to information obtained firsthand, from their own experience, in this case, than to clearly contradictory testimony. Although this type of situation is not representative of natural testimonial learning contexts (where those transmitting the information tend to do so without "jokes" or ill intent), it is sometimes true that the individual's intuitive knowledge may clash with the information provided by others. For example, testimony that the Earth is round or that air has weight contradicts ideas that are embedded in our intuitive experience. Findings show that children's acceptance of the testimony of others when this runs counter to their own intuition depends, among other things, on knowledge of the subject attributed to the informant. For example, Lane and Harris (2015) presented 3-8-year-old children with two informants who were experts in different fields (one, an animal expert; and the other, an artifact expert). The children selectively trusted one or other of the experts according to the type of task presented, either animal related or artifact related.

As well as playing a key role in the construction of knowledge, the testimony of others also serves to mitigate the ambiguity or uncertainty about some event (what has occurred?) or object (what is it?). In this type of context, the individual lacks information to assess or interpret a situation more fully. For example, Enesco, SebastiánEnesco, Guerrero, Quan, and Garijo (2016) presented 4-6-year olds a series of ambiguous peer interaction situations that could be interpreted in two different ways, either as an accident (e.g., the child fell off the swing) or as a malicious action (e.g., the child was pushed off the swing). Given these situations, the children were willing to trust the testimony of others in order to interpret what had happened. Furthermore, according to Morgan et al. (2015), from the age of 5 to 6 years, children assess their own level of uncertainty when faced by a task and compare this with testimonial information, before deciding whether to adopt the decisions of informants. This tendency has been called the "copy when uncertain" bias and, from an evolutionary perspective, is posited as one of the mechanisms responsible for the transmission of cultural knowledge across human evolution (Boyd \& Richerson, 1988; Claidière \& Whiten, 2012). Interestingly, this tendency to endorse testimonial information seems to be modulated by the valence of the information transmitted. Children (particularly preschoolers) are more sensitive to others' testimony when the information conveyed contains positive elements (e.g., nice opinions or assessments about third persons) than when it conveys negative elements (Boseovski \& Lee, 2008; Boseovski, Marble, \& Hughes, 2017). Thus, at least in attributional contexts, young children seem to be more influenced by a general positivity bias than by a tendency to copy when uncertain.

Thus far, we have reviewed the role of testimony in situations where individuals have different levels of certainty about the testimonial content (from ignorance to robust prior ideas). We have seen that children are much more likely to accept information that does not run counter to their own experience, or which serves to clarify ambiguous situations, compared to clearly contradictory or counterintuitive information. This is largely true regardless of the degree of consensus among the informants.

However, the few studies carried out in cultures other than Anglo-Saxon countries do not always confirm this general trend, and sometimes yield contrasting findings. For instance, Enesco et al. (2016) found that a majority of Chinese children accepted the criteria of a group of teachers who unanimously supported the exclusion of a peer (e.g., not letting a child play with the rest of the group) whereas Spanish children of the same age maintained their opposition to the exclusion, going against the teachers' unanimous opinion. However, interestingly, the children from both countries condemned the situation in the absence of testimonial information (Enesco et al., 2016). The authors suggested that the difference between the Spanish and Chinese children might be a result of culturespecific patterns related to children's attitudes toward adults, with Chinese children being more likely to be deferential than their Spanish counterparts. In the same line, using one of Asch's visual perception tasks, Corriveau, Kim, Song, and Harris (2013) found that Asian-American children were more sensitive to (or conformist with) consensus information provided by adults than were Caucasian-American children.

It could be tempting to interpret these findings in the light of the classic differentiation between collectivist and individualist cultures (Triandis, 1995). However, other studies have found a different relationship between children's cultural background and conformism. For example, Chan and Tardif (2013) compared 6-8-year olds 
from Hong Kong and the United States on an object classification task with objects of varying ambiguity, after hearing the opinion of a fictitious teacher. Although both the Chinese and American 8-year olds rejected unexpected labels for objects (i.e., wrong names) proposed by the teacher, only the Chinese 6-year olds kept to their own opinion whereas the American 6-year olds were more likely to endorse the teacher's label (see, however, Chen et al., 2013, for contrasting results). The authors interpreted these findings as an example of the difference in the metacognitive development of Chinese and American children, specifically the ability to identify how certain they feel about their own knowledge and their ability to use this information to decide whether to accept the testimony of others. According to Chan and Tardif (2013), in Chinese culture, more importance is attached to early autonomy and self-reliance compared to American culture, which would explain the difference between the 6-year olds from the two countries. Overall, the above findings suggest, at the very least, that the role of culture in testimonial learning cannot be simplistically reduced to belonging to supra-entities such as the so-called collectivist versus individualist cultures. Quite to the contrary, the role of culture should be understood under the framework of variables such as the field of knowledge in question, characteristics of the informants, participants' ages, and the values and educational practices of the particular cultural communities (see Fischer \& Poortinga, 2018 for an interesting discussion on culture-comparative research). In any event, further research is needed to elucidate how different cultural contexts impact testimonial learning, and more specifically, on sensitivity to consensus and deference to authority.

The present study aimed to shed light on this question by evaluating the endorsement of a unanimous majority in Chinese and Spanish children aged 4-5 years. We targeted this age group because findings consistently show that by the age of four, children are already sensitive and selective to the information offered within the testimony experimental paradigm (see Harris et al., 2018). In contrast to most of the studies on testimonial information, here we presented a unanimous majority composed by children of the same age as the participants. The few works in this regard show that children are sensitive to consensus information provided by peers, and, in some cases, side with them even when their opinion is in conflict with the children's perceptual experience or the normative judgments about social events (Haun \& Tomasello, 2011; Kim, Chen, Smetana, \& Greenberger, 2016). This tendency to conform to other children's opinions seems to follow a social motivation, the aim of being accepted into the peer group, what is called normative conformity (Claidière \& Whiten, 2012). Nonetheless, when they need to learn something new (i.e., informative conformity, Claidière \& Whiten, 2012), children prefer adult informants to children (e.g., Jaswal \& Neely, 2006). This suggests that, from an early age, children use the informant's age in a flexible way, depending on the context of decision involved. For example, preschoolers choose adult informants to know about nutritional value of food but choose peer informants to know how a toy works (VanderBorght \& Jaswal, 2009).

In this study, we explored two different contexts of decision, but which are of equal importance in the natural process of testimonial learning: the context of object functions and that of social interactions. In both contexts, individuals learn from others, in an inherently social environment. However, the context of object functions forms part of the domain of social conventions (e.g., what an artifact is used for and how it is used) whereas the context of social interactions is part of the domain of sociomoral judgments (e.g., the actor's intentions and the consequences of the act). In general, children do not confuse these two domains and are significantly more inflexible in response to sociomoral transgressions than transgressions regarding conventional actions (Turiel, 1983).

In addition, with the aim of assessing the role of prior knowledge in the participants' decisions, we varied the degree of ambiguity or uncertainty of the situations presented. In the unfamiliar situation, novel artifacts (object function context) and strange events (social evaluation context) were presented, and 10 informants unanimously chose one of the two options. In the ambiguous situation, both the objects and the social events were open to two possible interpretations, and the 10 informants unanimously opted for one of these. In the familiar situation, the objects and events were clearly familiar and recognizable for the participants. In this case, all informants provided a counterintuitive interpretation, in conflict with "common sense."

With regard to our hypotheses, we expected to find an effect of the situation (level of ambiguity): children will be more likely to follow the majority in the situations open to different interpretations (ambiguous) or clearly 
unknown to them (unfamiliar) compared to situations of which they have robust prior knowledge (familiar). In this last situation, participants were expected to follow their own criteria and rejected the majority's counterintuitive opinion. Additionally, we expected to find a difference between the context of object function and social evaluation: children will be more inclined to endorse a majority counterintuitive opinion on object function than on social regulation norms.

Regarding the participants' cultures, given that findings on the influence of testimony across different cultures are in some cases inconsistent, we anticipated different possible results depending on the relative value that Chinese and Spanish children give to consensus and informants' age. If we take a crude collectivist view of East Asians, Chinese children should be more sensitive to consensus, and therefore more likely to endorse the information provided by the group of peers, as compared to the Spanish children (in line with the findings of a previous study using adult informants, Enesco et al., 2016). However, it also may be that Chinese children give little credit to peers as informants, and so they are scarcely influenced by the pressure of consensus. Because this latter hypothesis does not exclude the Spanish children, a possible outcome would be that children from both countries respond similarly.

\section{2 | METHOD}

\section{1 | Participants}

A total of 108 preschoolers from China and Spain participated in the study. The Spanish sample comprised 48 participants ( $M=59.33$ months, Range $=43-70$ months, 24 females). The data were collected at a school in the province of Ciudad Real (Spain). The Chinese sample consisted of 60 participants ( $M=55.73$ months, Range $=$ 46-61, 30 females) from a school in the province of He Nan (China) (for datasets see Sebastián-Enesco, Guerrero, \& Enesco, 2019). Participants from both countries belonged to a medium socioeconomic status and lived in safe and industrialized neighborhoods (SES was not assessed individually, but by the school area). All participants from both countries attended school regularly since at least the age of three. Schools chosen in both countries were similar in terms of methodology and classroom organization following a traditional education system. Therefore, children were used to the school settings and to non-familiar adults (non-relatives), as well as to devices such as laptops and mobiles. No child had any learning disability, health problems, or atypical developmental circumstances. Children's and parents' native tongue was Spanish and Chinese, respectively. Written permission was obtained from the schools and all the participants' parents, and children gave their verbal consent before the study was initiated.

\section{2 | Design}

The task was administered individually on a computer in a quiet room facilitated by the schools. A Chinese speaker experimenter, born and raised in China and, therefore, familiar to the cultural background, collected the data from the Chinese participants. A Spanish speaker experimenter, born and raised in Spain and familiar to the cultural background, was responsible for collecting data from the Spanish children. The procedure was elaborated in Spain with the active help of the Chinese experimenter (who was a graduate student in Psychology in Madrid) in order to make all the materials and questions appropriate for the Chinese preschoolers. Moreover, both in Spain and China these materials were piloted with children of similar ages.

The procedure was exactly the same for the Spanish and Chinese children, with the exception of the characters who appeared in the task (drawings and photos, see Appendix A and Tables S1 and S2), who belonged to the same ethnic group as the participants, and the pseudowords used, which were adapted to the participants' language, Chinese or Spanish. All the experimental trials were structured in the same way: firstly, the situation 
and the two possible responses were presented, after which the participant was informed of the choice of a group of 10 child informants, who had all given the same response (unanimous consensus). Finally, the participant was asked to select an option.

\section{3 | Procedure}

Before the tasks were presented, the experimenter ( $E$, hereinafter) explained that she was visiting different schools to play a computer game with children of the same age. E told the participants that before answering each question, she was going to provide the response given by other girls and boys from different schools, and that once the participants had played the game, they would also tell the next children about the choices they had made. To this end, the photos of 10 children, 5 males and 5 females, supposedly corresponding to the previous participants were shown on the computer, together with a blank space where the photo of the present participant would be included for the following "players" (see Appendix A).

Before initiating the experimental phase, and to familiarize the participant with the procedure, two trials were administered in which the participant was shown a familiar object (e.g., a small, round car) and two possible labels for the object (car/ball), see Table S1. The participant was then asked to identify the correct name. No informants were presented in these familiarization trials. The aim of this phase was to ensure the participants understood they were expected to provide the correct information throughout the task. Immediately afterward, the tasks were initiated (for a detailed description of the procedure, see Appendix A).

For each situation (unfamiliar, ambiguous, familiar) within each context (object function, social interaction), two different trials were presented. Consequently, a total of 12 trials were administered. The order of the trials was counterbalanced considering the following criteria: (a) the familiar trials were always presented last to avoid a starting bias against the informants as a result of the counterintuitive opinions provided in response to the familiar situations; (b) the first trial was always an unfamiliar object function, the most neutral in nature, and thus less likely to bias the following children's responses. For the sake of simplicity, we created four different orders that were presented randomly across participants.

\subsection{1 | Decision contexts}

\section{Social evaluation}

Unfamiliar. In the unfamiliar trials, we presented novel situations adapted from Smetana (1985) consisting of an alien creature with a sad expression. In one trial, E explained that, on a distant planet, some aliens were "mibeando" (a Spanish pseudoword, in the form of a verb in gerund, in Chinese, "La Vei") another alien, so the pictured alien consequently felt sad. E then explained that she did not know whether it was right or wrong to "mibear." $\mathrm{E}$ then said that all the child informants whose photos appeared on the screen had said that it was OK to "mibear." The participant was then immediately asked, "What do you think? Is it right or wrong to mibear?" The other trial was exactly the same with the exception of the picture of the alien and the pseudoword used: dasclar (in Chinese, "Yi Shui") (see Table S2). Ambiguous. In these trials, we used the same material as in the study by Enesco et al. (2016), based in turn on the work by Killen, Sinno, and Margie (2007). In one trial, a drawing was presented of two children in a schoolyard. One of them (e.g., María, in Spanish) was sitting on the ground in front of a swing and the other (e.g., Cristina, in Spanish) was standing behind the swing. E told the participant she did not know whether María had fallen accidentally or Cristina had intentionally pushed her off the swing. The participant was told that all the informants had interpreted the situation as an accident (e.g., "María fell off"). The participant was then asked to indicate their interpretation. The other trial consisted of a picture of two more children in a similar situation in front of a slide: the main character might have fallen or been pushed off by the other. Again, the informants unanimously interpreted the situation as an accident, and the participant was asked the same question as before. The situations presented here were tested previously in a pilot study to ensure that any interpretation was equally 
plausible (see Enesco et al., 2016). Familiar. We presented two social exclusion situations, based on the ones used in the study by Enesco et al. (2016). A pilot study showed that Spanish and Chinese children consistently condemned these social exclusion situations (see Enesco et al., 2016). In one of the trials, the participant was shown a drawing of three children playing football in the schoolyard and another child sitting to one side with a sad expression on her face. E explained that the group of three children had not let the lonely child (Hong-Hong, in Chinese) join in the game. Then, she explained that all the child informants had said it was right not to let Hong-Hong play. Finally, the participant was asked, "Is it right or wrong not to let Hong-hong play?" The other trial consisted of a similar social exclusion situation but in the context of a game of basketball. Likewise, all the informants had approved the social exclusion, and the participant was asked the same question.

\section{Object function}

Unfamiliar. We presented pictures of made-up unfamiliar objects. In one trial, E explained that the object was used to make holes in the ceiling or in the floor but she did not know which of the two. In the other trial, E said the unfamiliar object could be used to clean the floor or walls, but, again, she did not know which. After each trial, E explained what the 10 informants had said (the former object was for making holes in the ceiling, and the latter for washing the floor; see Table S3). Ambiguous. We presented two pictures of ambiguous objects, similar to those used in the study by Chan (2011). One of them could have been a lamp or a chair; the other, gloves or socks. E told the participants she did not know what the object in question was for, explaining the two alternatives (e.g., "They might be to put on your hands or your feet."). The group of informants had unanimously selected the options of lamp and socks, respectively. Familiar. A familiar object was presented, an everyday table in one trial and a clothes peg in the other. E then told the participants that the object was used for its original function (to eat at, and to hang out washing, respectively), or for an alternative function (to lie down on, or to hold your hair in place, respectively). The informants chose the alternative function in both trials.

After the tasks had been administered, children were asked a series of control memory questions to ensure that they had paid attention to the tasks. E displayed again, one by one, 6 of the 12 trials presented during testing, and asked the participants if they remembered (a) which option they had chosen, (b) which option the 10 informants had chosen, see Appendix A. Immediately after, E thanked the participants for their participation and debriefed them.

\section{4 | Coding}

Our main dependent variable was the endorsement of the majority view. We coded 1 if the participants chose the option provided by the majority, and 0 if they opted for the alternative. The experimenter registered the participants' choices in situ.

\section{3 | RESULTS}

Drawing on the statistical approach implemented in Enesco et al. (2016), we used the following categorical tests: Binomial tests to determine whether the participants from each cultural community endorsed the majority view above chance level; chi-square tests to compare choices between Spanish and Chinese children; McNemar tests for comparisons between contexts of decision (object function and social evaluation) and situations (unfamiliar, ambiguous, and familiar). Firstly, we present the general trends of children's overall endorsement of the majority view. For this, we computed the total number of choices siding with the majority for each participant across the 12 trials. Secondly, we focus on the endorsement of the majority view within each context and situation, comparing the Chinese and Spanish children's choices in each case. Finally, we explore the children's memory of their own and the informants' choices in 6 out of the 12 trials. We coded 1 if they remembered correctly and 0 if they did 
not remember or they remembered incorrectly. For each participant, we computed two total scores, one regarding their own choices (self-memory), and the other regarding the informants' choices (others memory).

The preliminary analyses showed no gender differences in children's general endorsement of the peer majority, Mann-Whitney test $U=.667, p=.505$. Similarly, we found no significant effect of the age (in months) of participants (rs $(108)=.012, p=.898$ ) or the order of trial presentation (Kruskal-Wallis test $H(3)=2.37, p=.499)$ on overall trust in the majority. Hence, none of these variables were included in the following analyses. With regard to the familiarization trials, the majority of participants correctly identified the object presented in Trial 1 (98.1\%) and Trial 2 (96.3\%).

In line with our predictions, the level of ambiguity influenced the general tendency to follow the majority, chisquare test $\chi^{2}(3, N=1,296)=159.4, p<.001$. Pair-wise comparisons using continuity-corrected McNemar tests with Bonferroni correction showed participants were more willing to endorse the majority option in unfamiliar (67.1\%) and ambiguous situations (71.1\%) compared to familiar situations $(32.4 \%)(p<.001)$, but no differences were found between the ambiguous and unfamiliar situations ( $p>$.017). Regarding the familiar situations, the participants were overall more willing to side with a majority that supported a counterintuitive view of a familiar object function (familiar object function situation: $40 \%$ of responses) than a majority that supported a counterintuitive view on a social regulation norm (familiar social evaluation situation: $25 \%$ of responses), McNemar test $p<.001$. Finally, in general terms, the Spanish participants were significantly more willing to follow the peer majority (65.8\%) than the Chinese participants (49.7\%), chi-square test, $\chi^{2}(2, N=1,296)=33.7, p<.001$.

\section{1 | Social evaluation domain}

\subsection{1 | Unfamiliar}

In the unfamiliar situation, both the Spanish children (binomial test: $65.6 \%$ of responses, $p=.003$ ) and the Chinese children (binomial test: $65.8 \%$ of responses, $p=.001$ ) were significantly more inclined to follow the peer majority view (e.g., it is right to "mibear"/"La Vei") compared to the alternative option (it is wrong), with no significant differences between countries, chi-square test $\chi^{2}(2, N=216)=.001, p=.974$.

\subsection{2 | Ambiguous}

Both the Spanish and Chinese participants backed the interpretation chosen by the majority (she has fallen off) beyond chance level, binomial test: China $p=.008$; Spain $p<.001$. However, the Spanish children $(85.4 \%$ of responses) endorsed the majority option significantly more often than their Chinese counterparts (62.5\% of responses), chi-square test $\chi^{2}(2, N=216)=14.11, p<.001$.

\subsection{3 | Familiar}

When a familiar situation was presented to participants, both the Spanish children ( $21.1 \%$ of responses endorsing the majority) and the Chinese children (23.3\% of responses endorsing the majority) chose the alternative option, in line with their prior ideas, binomial test $p<.001$. Moreover, no differences were found between the Chinese and Spanish participants, chi-square test $\chi^{2}(2, N=216)=.40, p=.527$.

In summary, the children from both cultural communities followed the peer majority when the situation was unfamiliar and opted for their own criteria when the situation was familiar to them. When the situation was open to different interpretations, both Spanish and Chinese children trusted in the majority opinion. However, the Spanish children were significantly more inclined to the peer majority view than the Chinese children (see Figure 1). 


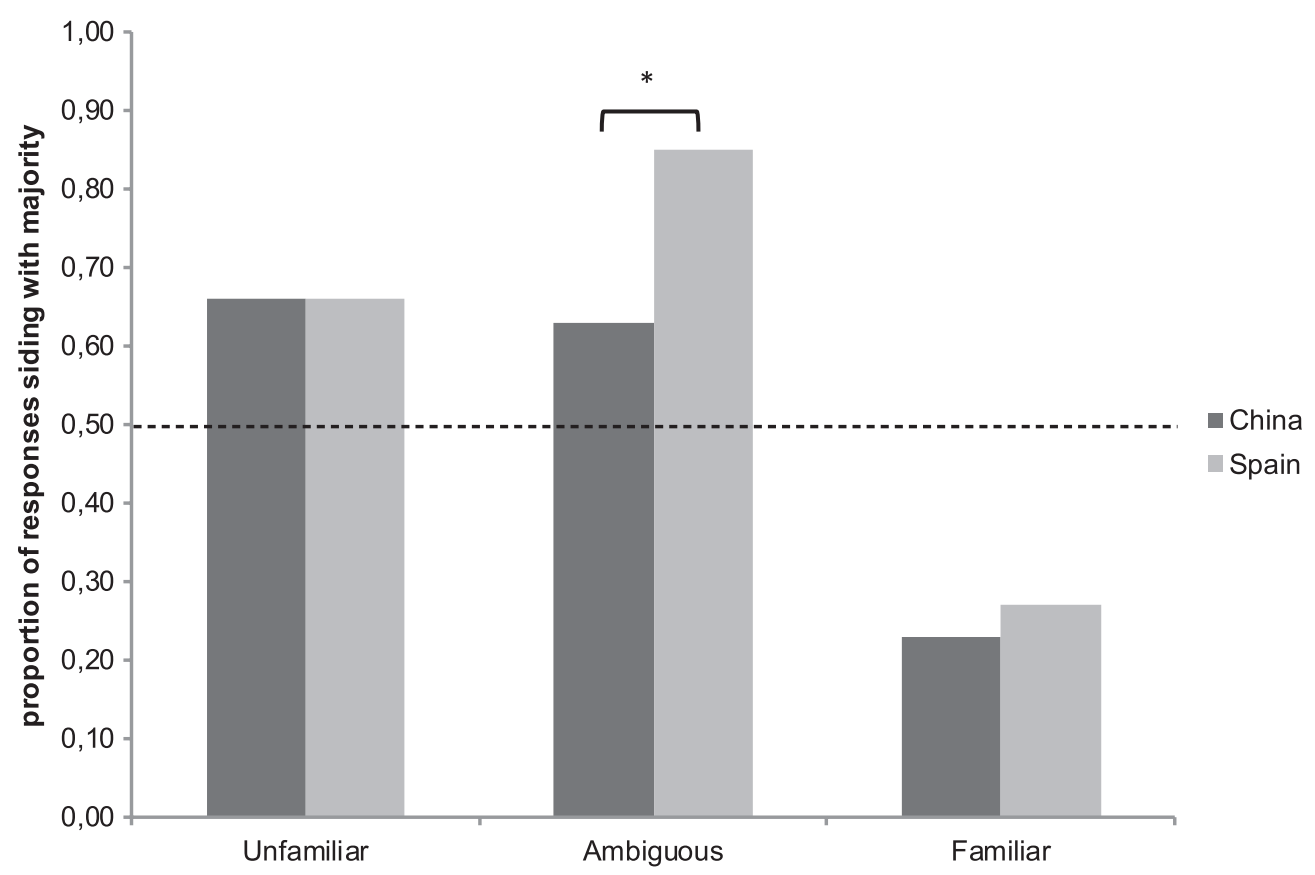

FIGURE 1 Proportion of responses siding with the majority in the social evaluation context for Chinese and Spanish participants

\section{2 | Object function domain}

\subsection{1 | Unfamiliar}

When deciding the potential use of an unfamiliar artifact, the Spanish children followed the majority (binomial test, $83.3 \%$ of responses, $p<.001$ ) whereas the Chinese participants showed no clear preference for either of the two options (binomial test, $56.7 \%$ of responses, $p=.171$ ). This difference between Chinese and Spanish children was highly significant, chi-square test $\chi^{2}(2, N=216)=17.58, p<.001$.

\subsection{2 | Ambiguous}

The Spanish participants consistently sided with the majority when the object presented could have had two functions (e.g., a lamp or a chair), binomial test $82.3 \%$ of responses, $p<.001$. In the case of the Chinese participants, the results revealed a marginally significant tendency to follow the majority, binomial test $59.2 \%$ of responses, $p=.055$. Again, the difference between countries in the majority endorsement was significant, chi-square test $\chi^{2}(2, N=216)=13.44, p<.001$.

\subsection{3 | Familiar}

The Chinese participants followed their own criteria rather than the majority opinion when a familiar object was presented, binomial test $30.8 \%$ of responses in favor of the majority, $p<.001$. However, in the Spanish children, the responses were equally distributed between the majority opinion and the alternative option, binomial test $51 \%$ of responses siding with the majority, $p=.919$. The difference between countries were again significant, chi-square test $\chi^{2}(2, N=216)=9.09, p=.003$. 
In short, in the object function domain, the Spanish children showed a consistent and strong tendency to follow the unanimous peer majority when the object was unfamiliar or ambiguous. Even in the case of familiar objects, a substantial percentage of responses endorsed the majority opinion, despite this clearly contradicting their prior knowledge. The Chinese children, however, resisted siding with the majority in this context, and even in the cases of unfamiliar and ambiguous objects, they showed no clear tendency to side with the unanimous majority (see Figure 2).

\subsection{Control memory checks}

Regarding the control memory questions presented at the end of the tasks, both Chinese and Spanish children showed high levels of performance when asked to remember their own choices (Spanish children: 85\% correct answers; Chinese children: $81 \%$ correct answers), and to a lesser extent, when asked about the informants' choices (Spanish children: 71\% correct answers; Chinese children: 66\% correct answers). Crucially, no differences were found between countries, chi-square tests, Self-memory: $\chi^{2}(2, N=647)=2.58, p=.108$; Others memory: $\chi^{2}(2, N=647)=1.77, p=.184$.

\section{4 | DISCUSSION}

The present study explored Chinese and Spanish preschoolers' endorsement of a unanimous peer majority in two different contexts: object function and social evaluation. In each context, three levels of ambiguity were used in the situation presented: unfamiliar, ambiguous, and familiar. Broadly speaking, the results revealed, as expected, that our participants were more willing to follow the majority in unfamiliar or ambiguous situations than in familiar ones. In line with previous studies (e.g., Einav, 2014), this finding suggests that children's acceptance of testimonial information strongly depends on their prior knowledge or beliefs. However, the results also show the influence of the context of decision in children's readiness to endorse a majority counterintuitive view. Thus, our participants

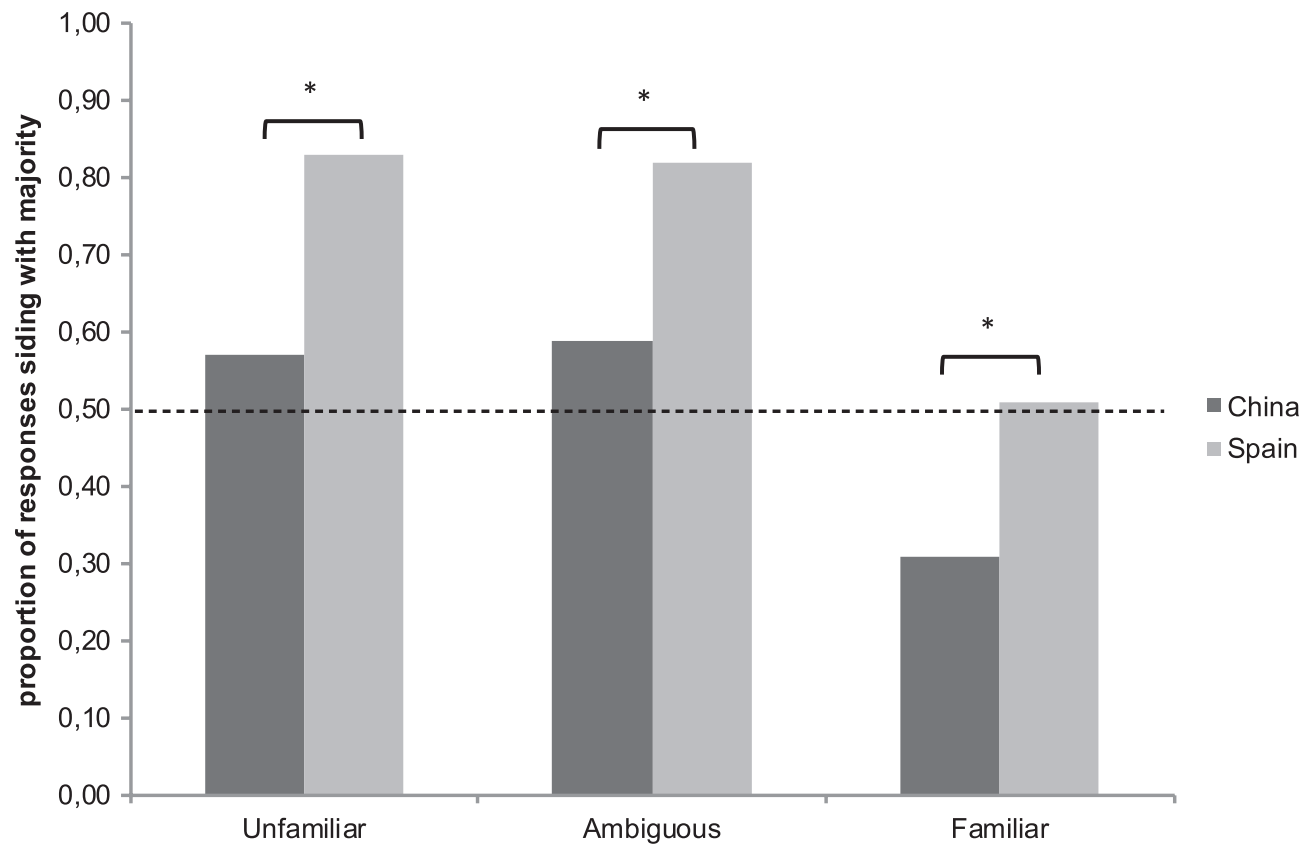

FIGURE 2 Proportion of responses siding with the majority in the object function context for Chinese and Spanish participants 
were significantly more inclined to accept a counterintuitive or non-normative opinion regarding the use of an object than regarding a peer exclusion event. This finding is consistent with expectations from the social domain theory view according to which children construct distinct systems of thinking in the domains of moral, conventional, and personal judgments (Nucci, 2001; Smetana, 1985; Turiel, 1983). Although the paradigm used here is different from the one normally used in the field of social moral reasoning, our participants' choices across the trials suggest that they perceived conventions to be more modifiable than moral norms. Within a social conformity paradigm, Kim and colleagues (2016) also found that children were much less susceptible to peer pressure when the issue dealt with hitting or teasing another child than when it had to do with unconventional actions (e.g., wearing a bathing suit to day care).

Finally, in contrast to our expectations, the Spanish children followed the majority significantly more often (65.8\%) than their Chinese counterparts (54.5\%). This finding contrasts with the results of the previous study with comparable samples of Spanish and Chinese children (Enesco et al., 2016), which used adults as informants rather than peers. In that study, the Chinese participants tended to endorse the unanimous majority's opinion significantly more (88.2\%) than the Spanish children (79.3\%). This general comparison should be treated with caution due to the methodological differences between the two studies (e.g., some of the tasks were different; in the present study, the majority consisted of 10 children whereas the informants in the previous study were 3 adults; in the previous study, 6 trials were administered and in the present study, 12). Nonetheless, two of the tasks used by Enesco et al. (2016) are directly comparable to the familiar and ambiguous social evaluation situations presented in the current study. The familiar situation consisted of a social exclusion event in which a group of children would not let another child take part in a game: The unanimous majority, made up of adult informants in the previous study and children in the present one, approved of the apparent moral transgression. In this situation, the Spanish children were equally reluctant to side with the majority endorsing the social exclusion, regardless of whether the majority comprised adults or peers (21\% of endorsement in both cases). In contrast, the Chinese children, who widely rejected a unanimous peer majority that supported the exclusion of a peer, (only $23 \%$ followed the majority in the present study), accepted the exclusion when it was supported by a unanimous adult majority (65\%). In other words, whereas the Spanish children were reluctant to accept a peer exclusion event whoever endorsed it, the Chinese accepted it if it was sanctioned by a group of adults.

In the ambiguous situation, consisting of a social situation open to two interpretations, both the adult majority and the child majority unanimously interpreted it in the same way. Most of the Spanish participants endorsed the informants' interpretation, regardless of whether they were adults (80\% followed the majority) or children (85\%). In contrast, the Chinese participants were significantly more inclined to follow the majority composed of adults (80\%) compared to peers (62\%). Once more, it seems that when the interpretation of a social interaction is uncertain, the Spanish children trusted the majority regardless of the informant type whereas the Chinese children were more likely to do so when the informants were adults rather than children.

Tentatively, these findings suggest that Chinese and Spanish children adopt different views of the relation between the source-who informs-and the content-what they say. The Spanish participants appear to be guided fundamentally by the content transmitted by others: if they lack sufficient information to assess a social situation, they trust the majority, but, in contrast, if they already have their own criteria, these ideas prevail over the testimonial information, whoever provided it. The Chinese participants appear to focus more on the informants' identity and give less weight to their own prior ideas when the information is provided by a group of adults. This does not mean that the Chinese children are blind followers of adult opinion and suspend their own criteria in favor of that of adults. Quite possibly, they infer that if an adult majority approves of a child's exclusion, there may be important reasons to do so (e.g., the child is too small to play the game and might get hurt). Several studies on children's reasoning about social exclusion have provided evidence that is coherent with this interpretation. Killen, Pisacane, Lee-Kim, and Ardila-Rey (2001) found, for example, that American children accepted or not the social exclusion of a peer depending on whether they adopted a moral perspective (e.g., exclusion based on race is wrong) or an organizational perspective (e.g., exclusion due to the lack of space is acceptable). In addition, 
Fang et al. (2003) showed that Chinese children were more prone to accept apparently arbitrary decisions from parents compared to American children, and tended to ascribe reasons to make sense of the decision. In line with these findings, it is possible that our Chinese participants, in response to an apparently unconventional opinion provided by knowledgeable individuals, such as adults, look for further reasons that might justify this decision. In contrast, Spanish children, may be not concerned about finding alternative explanations, but primarily focus on the situation itself.

Furthermore, our findings suggest that the Chinese children give little credibility to their peers' testimony, at least in the epistemic domains addressed herein. This is particularly notable in the unfamiliar and ambiguous object function contexts, in which, when presented with unusual artifacts, individuals are more likely to accept the claims of others. Not even in these cases did the Chinese children clearly opt for the peer-majority's interpretation. Unfortunately, we have no data on the reasons that might have led them to go against their peers' testimony and, to the best of our knowledge, no studies have addressed the perceived epistemic status of other children in Chinese population. It may well be that Chinese children preferentially perceive adults as reliable sources of knowledge more than their Western counterparts, investing their peers with other types of functions, such as a social role (i.e., more related to the regulation of interpersonal relations between peers). In fact, the previously cited study by Fang et al. (2003) showed that, in contrast to Western children, Chinese children gave greater value to a mother's knowledge of a child's needs than to that of the child herself. Moreover, a recent report on Chinese adults' perceptions of parenting and child behavior across generations show that nowadays Chinese parents still perceive the influence of peers as a potential risk in their children's personal success, and therefore, they are expected to restrict their young children's choices of friends (Zhou, Yiu, Wu, \& Greenfield, 2018, see also Tamis-LeMonda et al., 2008).

This tendency to call into question their peers' claims clearly contrasts with the Spanish children's decisions. When the peer majority proposed an alternative function for a familiar object (e.g., a table is for lying down on), the Spanish children were less inclined than their Chinese peers to follow their own criteria, and half of them sided with the majority opinion. Although we have no data that are directly comparable with adult informants, in previous research, a series of teacher informants proposed alternative functions for familiar objects (Guerrero et al., 2017) (e.g., a clean, new sand bucket for serving a salad) and a sample of Spanish preschoolers were asked to evaluate the situation. A large majority of them opposed this novel interpretation, even when the testimonial source was their own teacher or a unanimous majority of fictitious teachers. It seems, then, that in the object function domain, in contrast to that of social evaluation, Spanish children defer to some extent to their peers, but this deference does not extend to adult informants. Although, due to our study design, we cannot explore the processes underlying this specific pattern of conformity, we can posit some explanations. Arguably, if a peer group proposes alternative functions for objects, Spanish children interpret this as a type of game, as a fun way to answer, obviating any epistemic context. However, this is not so when the children are in a school setting and the testimonial source are teachers, as was the case in the study by Guerrero et al. (2017). This context may have encouraged the participants in Guerrero and colleagues' study to adopt an epistemic stance of the teachers' opinion (interpreted as a kind of mistake, or as a "trick" to assess children's conventional knowledge). In a recent study on the selective learning of new words (Henderson, Graham, \& Schell, 2015), young children were found to trust an informant who, in a previous phase of the experiment, used a familiar object in a non-conventional way (e.g., using a spoon to comb their hair), but not when the same informant made mistakes with the labels of familiar objects (e.g., calling a spoon a comb). According to the authors, this suggests the children interpreted the "strange" actions in the first case as a type of game, and thus, they did not diminish the informant's credibility. In contrast, in the second case, the actions were directly evaluated epistemically, leading the children to resist learning the words proposed by the informant.

In line with these findings, we suggest that, compared to their Chinese counterparts, the Spanish children in our study were more prone to seek an alternative interpretation for the atypical testimony of a peer group. This, in turn, might be explained by the Spanish children's greater sensitivity toward peer influence. From 4 to 5 years of age, peers begin to act as a reference group, defining values and other types of regulatory norms (Dunn, 1988). 
This typically encourages solidarity and uniformity among the members of the peer group, and helps define the identity of a group of children. In Spain, as in other Mediterranean countries, personal well-being strongly depends on social relationships, especially friendships (Requena Santos, 1994). Indeed, studies in Spain have shown adolescents and pre-adolescents to be more oriented toward peer affiliation than in other European societies (e.g., Buchmann \& Dalton, 2002; Requena Santos, 1994). For example, the value a group of friends attaches to academic performance is a much more powerful predictor of academic success in Spanish teenagers than in adolescents in Switzerland, France, and other European countries (Buchmann \& Dalton, 2002). The notion of honor among Spanish adolescents is intimately linked to behaviors that promote interdependence with others such as being generous or fulfilling one's social obligations, which is not the case in Northern European countries, where honor tends to be defined by individual variables (Mosquera, Manstead, \& Fischer, 2002). Although we have not been able to find comparative studies with young children, these findings, overall, seem to support the hypothesis that Spanish children take their peers' opinions into account more than in other societies, such as the Chinese.

In summary, the findings of the present study, together with those by Enesco et al. (2016), suggest that preschoolers from different cultural communities are sensitive to consensus information and even prioritize it on occasions over their own criteria. Nonetheless, the informants' age (adults or peers) seems to have a different impact on Chinese and Spanish children, depending on the domain under study. Peers appear to exert greater social pressure on the Spanish participants, provided they do not contradict basic aspects of moral norms. For Chinese children, however, peers seem to have little authority (either epistemic or normative), in contrast to adults, to whom they are significantly deferential, even when their opinions appear to go against established norms (Enesco et al., 2016). In our study, we speculate on the reasons that might have led the children from both communities to follow (or not) the opinion of a unanimous majority. Crucially, the cultural differences found here were not likely caused by differences regarding attentional processes, as shown by the control memory checks. Unfortunately, our research cannot elucidate the specific aspects of the educational practices and social values in each community that affect the children's decisions and, more importantly, the attitudes they activate in response to the testimony of others. Any comparison between cultural groups must be very cautious when it comes to interpreting the results. Being aware of this limitation from the very beginning, we selected two samples that were as similar as possible in terms of general sociodemographic variables and school educational system. However, these similarities may conceal subtle differences between Chinese and Spanish pupils in other aspects relevant to this research. For example, the degree in which peers communicate with each other in the classroom for different purposes (e.g., sharing information, joking or teasing each other) as well as the teachers' reactions to these behaviors may vary substantially between countries. Such differences in the classroom climate could be at the root of children's different orientation toward peers' testimonies.

Any real advance in the field of cultural psychology requires a careful analysis of the different contexts and levels of experience where a certain cultural process takes place (Fischer \& Poortinga, 2018). On a daily basis, individuals face situations that evoke conflicting social values, traditionally identified as "individualist" and "collectivist" (Tamis-LeMonda et al., 2008). Therefore, culture-comparative research needs to abandon the essentialist conception of "cultures" as categorical and static entities.

\section{ACKNOWLEDGMENTS}

We owe thanks to Siyu Quan and Cristina Cascado for their assistance in data collection in China and Spain, respectively, and to María Núñez and the anonymous reviewers for helpful comments on the manuscript. We also thank the school centers and all participants for their cooperation.

\section{CONFLICT OF INTEREST}

All authors declare no conflicts of interest. 


\section{DATA AVAILABILITY STATEMENT}

The data that support the findings of this study are openly available in figshare at https://doi.org/10.6084/m9.figsh are.7901852.v1 [dataset] Sebastián-Enesco, Carla; Guerrero, Silvia; Enesco, lleana; 2019; Data_Sebastián-enesco et al..xls. figshare; https://doi.org/10.6084/m9.figshare.7901852.v1

\section{ORCID}

Carla Sebastián-Enesco iD https://orcid.org/0000-0001-7340-0038

\section{REFERENCES}

Asch, S. E. (1956). Studies of independence and conformity: A minority of one against a unanimous majority. Psychological Monographs, 70, 1-70. https://doi.org/10.1037/h0093718

Bernard, S., Harris, P. L., Terrier, N., \& Clément, F. (2015). Children weigh the number of informants and perceptual uncertainty when identifying objects. Journal of Experimental Child Psychology, 136, 70-81. https://doi.org/10.1016/ j.jecp.2015.03.009

Bond, R., \& Smith, P. B. (1996). Culture and conformity: A meta-analysis of studies using Asch's (1952b, 1956) line judgment task. Psychological Bulletin, 119, 111-137. https://doi.org/10.1037//0033-2909.119.1.111

Boseovski, J. J., \& Lee, K. (2008). Seeing the world through rose-colored glasses? Neglect of consensus information in young children's personalityjudgments. Social Development,17,399-416.https://doi.org/10.1111/j.1467-9507.2007.00431.x

Boseovski, J. J., Marble, K. E., \& Hughes, C. (2017). Role of expertise, consensus, and informational valence in children's performance judgments. Social Development, 26, 445-465. https://doi.org/10.1111/sode.12205

Boyd, R., \& Richerson, P. J. (1988). An evolutionary model of social learning: The effects of spatial and temporal variation. In T. R. Zentall \& B. G. Galef (Eds.), Social learning: Psychological and biological perspectives (pp. 29-48). Mahwah, NJ: Lawrence Erlbaum.

Buchmann, C., \& Dalton, B. (2002). Interpersonal influences and educational aspirations in 12 countries: The importance of institutional context. Sociology of Education, 75, 99-122. https://doi.org/10.2307/3090287

Chan, C. C. Y. (2011). The role of prior belief in children's trust in testimony: A social ecological approach (Doctoral dissertation). Retrieved from ProQuest Dissertations and Theses http://deepblue.lib.umich.edu/bitstream/handl e/2027.42/84497/chancc_1.pdf?sequence=1 (UMI No. 3458832).

Chan, C., \& Tardif, T. (2013). Knowing better: The role of prior knowledge and culture in trust in testimony. Developmental Psychology, 49, 591-601. https://doi.org/10.1037/a0031336

Chen, E. E., Corriveau, K. H., \& Harris, P. L. (2013). Children trust a consensus composed of outgroup members-But do not retain that trust. Child Development, 84, 269-282. https://doi.org/10.1111/j.1467-8624.2012.01850.x

Claidière, N., \& Whiten, A. (2012). Integrating the study of conformity and culture in humans and nonhuman animals. Psychological Bulletin, 138, 126-145. https://doi.org/10.1037/a0025868

Corriveau, K. H., Fusaro, M., \& Harris, P. L. (2009). Going with the flow preschoolers prefer nondissenters as informants. Psychological Science, 20, 372-377. https://doi.org/10.1111/j.1467-9280.2009.02291.x

Corriveau, K. H., Kim, E., Song, G., \& Harris, P. L. (2013). Young children's deference to a consensus varies by culture and judgment setting. Journal of Cognition and Culture, 13, 367-381. https://doi.org/10.1163/15685373-12342099

Dunn, J. (1988). The beginnings of social understanding. Cambridge, MA: Harvard University Press.

Einav, S. (2014). Does the majority always know best? Young children's flexible trust in majority opinion. PLoS ONE, 9, e104585. https://doi.org/10.1371/journal.pone.0104585

Enesco, I., Sebastián-Enesco, C., Guerrero, S., Quan, S., \& Garijo, S. (2016). What makes children defy majorities? The role of dissenters in Chinese and Spanish preschoolers' social judgments. Frontiers in Psychology, 7, 1695. https://doi. org/10.3389/fpsyg.2016.01695

Fang, G., Fang, F., Keller, M., Edelstein, W., Kehle, T., \& Bray, M. (2003). Social moral reasoning in Chinese children: A developmental study. Psychology in the Schools, 40, 125-138. https://doi.org/10.1002/pits.10074

Fischer, R., \& Poortinga, Y. H. (2018). Addressing methodological challenges in culture-comparative research. Journal of Cross-Cultural Psychology, 49, 691-712. https://doi.org/10.1177/0022022117738086

Guerrero, S., Cascado, C., Sausa, M., \& Enesco, I. (2017). My teacher is wrong: Preschoolers' opposition to nonconventional statements. Early Childhood Research Quarterly, 39, 1-13. https://doi.org/10.1016/j.ecresq.2016.11.001

Harris, P. L., Koenig, M. A., Corriveau, K. H., \& Jaswal, V. K. (2018). Cognitive foundations of learning from testimony. Annual Review of Psychology, 69, 251-273. https://doi.org/10.1146/annurev-psych-122216-011710 
Haun, D. B., \& Tomasello, M. (2011). Conformity to peer pressure in preschool children. Child Development, 82, 17591767. https://doi.org/10.1111/j.1467-8624.2011.01666.x

Henderson, A. M., Graham, S. A., \& Schell, V. (2015). 24-month-olds' selective learning is not an all-or-none phenomenon. PLoS ONE, 10, e0131215. https://doi.org/10.1371/journal.pone.0131215

Jaswal, V. K., \& Neely, L. A. (2006). Adults don't always know best: Preschoolers use past reliability over age when learning new words. Psychological Science, 17, 757-758. https://doi.org/10.1111/j.1467-9280.2006.01778.x

Killen, M., Pisacane, K., Lee-Kim, J., \& Ardila-Rey, A. (2001). Fairness or stereotypes? Young children's priorities when evaluating group exclusion and inclusion. Developmental Psychology, 37, 587-596. https://doi.org/ 10.1037/0012-1649.37.5.587

Killen, M., Sinno, S., \& Margie, N. G. (2007). Children's experiences and judgments about group exclusion and inclusion. Advances in Child Development and Behavior, 35, 173-218. https://doi.org/10.1016/B978-0-12-009735-7.50010-4

Kim, E. B., Chen, C., Smetana, J. G., \& Greenberger, E. (2016). Does children's moral compass waver under social pressure? Using the conformity paradigm to test preschoolers' moral and social-conventional judgments. Journal of Experimental Child Psychology, 150, 241-251. https://doi.org/10.1016/j.jecp.2016.06.006

Lane, J. D., \& Harris, P. L. (2015). The roles of intuition and informants' expertise in children's epistemic trust. Child Development, 86, 919-926. https://doi.org/10.1111/cdev.12324

Morgan, T. J., Laland, K. N., \& Harris, P. L. (2015). The development of adaptive conformity in young children: Effects of uncertainty and consensus. Developmental Science, 18, 511-524. https://doi.org/10.1111/desc.12231

Mosquera, P. M. R., Manstead, A. S., \& Fischer, A. H. (2002). Honor in the Mediterranean and Northern Europe. Journal of Cross-cultural Psychology, 33, 16-36. https://doi.org/10.1177/0022022102033001002

Nucci, L. P. (2001). Education in the moral domain. New York, NY: Cambridge University Press.

Requena Santos, F. (1994). Redes de amistad, felicidad y familia [Networks of friendship, happiness, and family]. Revista Española de Investigaciones Sociológicas, 66, 73-89. https://doi.org/10.2307/40183718

Schillaci, R. S., \& Kelemen, D. (2014). Children's conformity when acquiring novel conventions: The case of artifacts. Journal of Cognition and Development, 15, 569-583. https://doi.org/10.1080/15248372.2013.784973

Sebastián-Enesco, C., Guerrero, S., \& Enesco, I. (2019). Data_Sebastián-enesco et al. xls. figshare. https://doi.org/10.6084/ m9.figshare.7901852.v1

Smetana, J. G. (1985). Preschool children's conceptions of transgressions: Effects of varying moral and conventional domain-related attributes. Developmental Psychology, 21, 18-20. https://doi.org/10.1037/0012-1649.21.1.18

Tamis-LeMonda, C. S., Way, N., Hughes, D., Yoshikawa, H., Kalman, R. K., \& Niwa, E. Y. (2008). Parents' goals for children: The dynamic coexistence of individualism and collectivism in cultures and individuals. Social Development, 17, 183-209. https://doi.org/10.1111/j.1467-9507.2007.00419.x

Tomasello, M. (1999). The cultural origins of human cognition. Cambridge, MA: Harvard University Press.

Triandis, H. C. (1995). Individualism and collectivism. Boulder, CO: Westview Press.

Turiel, E. (1983). The development of social knowledge: Morality and convention. Cambridge, MA: Cambridge University Press.

VanderBorght, M., \& Jaswal, V. K. (2009). Who knows best? Preschoolers sometimes prefer child informants over adult informants. Infant and Child Development: An International Journal of Research and Practice, 18, 61-71. https://doi. org/10.1002/icd.591

Zhou, C., Yiu, W. Y. V., Wu, M. S., \& Greenfield, P. M. (2018). Perception of cross-generational differences in child behavior and parent socialization: A mixed-method interview study with grandmothers in China. Journal of Cross-Cultural Psychology, 49, 62-81. https://doi.org/10.1177/0022022117736029

\section{SUPPORTING INFORMATION}

Additional supporting information may be found online in the Supporting Information section at the end of the article.

How to cite this article: Sebastián-Enesco C, Guerrero S, Enesco I. What makes children defy their peers? Chinese and Spanish preschoolers' decisions to trust (or not) peer consensus. Social Development.

2019;00:1-15. https://doi.org/10.1111/sode.12416 